\title{
Optical and electronic properties of undoped La2Be2O5 single crystals in the far ultraviolet energy range
}

ARTICLE in JOURNAL OF THE OPTICAL SOCIETY OF AMERICA B · FEBRUARY 2015

Impact Factor: $1.81 \cdot$ DOI: 10.1364/JOSAB.32.000241

DOWNLOADS

23

3 AUTHORS, INCLUDING:

Igor N. Ogorodnikov

Ural Federal University

187 PUBLICATIONS 610 CITATIONS

SEE PROFILE
VIEWS

10 


\title{
Optical and electronic properties of undoped $\mathrm{La}_{2} \mathrm{Be}_{2} \mathrm{O}_{5}$ single crystals in the far ultraviolet energy range
}

\author{
Vladimir A. Pustovarov, ${ }^{1}$ Igor N. Ogorodnikov, ${ }^{1, *}$ and Erbol A. Ospanbekov ${ }^{2}$ \\ ${ }^{1}$ Experimental Physics Department, Ural Federal University, 19 Mira Street, Ekaterinburg, 620002, Russia \\ ${ }^{2}$ Kazakh National Pedagogical University named after Abai, \\ 13 Dostyk Avenue, Almaty, 050010, Republic of Kazakhstan
}

compiled: January 12, 2015

\begin{abstract}
The optical and electronic properties of undoped $\mathrm{La}_{2} \mathrm{Be}_{2} \mathrm{O}_{5}$ single crystals were determined using low-temperature $(T=10 \mathrm{~K})$ far ultraviolet (4.9-33 eV) synchrotron radiation spectroscopy, optical absorption spectroscopy ( $T=80$ and $320 \mathrm{~K}$ ) and calculations for the dispersions of the optical functions. On the basis of the obtained data we determined the Urbach's formula parameters and the cut-off energy $E_{\mathrm{c}}=5.49 \mathrm{eV}$ at $80 \mathrm{~K}$ for the low-energy tail of the host absorption, the bandgap $E_{\mathrm{g}}=6.78 \mathrm{eV}$ at $10 \mathrm{~K}$, the energy threshold for creation of unrelaxed excitons $E_{\mathrm{ex}}=6.28 \mathrm{eV}$, and other electronic properties.

OCIS codes: $\quad$ (160.2220) Defect-center materials; (160.3220) Ionic crystals; (160.3380) Laser materials; (160.4760) Optical properties.

http://dx.doi.org/10.1364/JOSAB.32.000241
\end{abstract}

\section{Introduction}

Lanthanum beryllate $\mathrm{La}_{2} \mathrm{Be}_{2} \mathrm{O}_{5}$ (BLO) is a popular optical material for many practical applications. The BLO: $\mathrm{Nd}^{3+}$ single crystals are known as highly efficient material for solidstate lasers oscillating at wavelength $1.07-1.08 \mu \mathrm{m}$. The output of BLO:Nd was shown to exceed that of YAG:Nd at low Q-switched repetition rates $(1 \mathrm{~Hz})$. For example, a Qswitched output of $450 \mathrm{~mJ}$ from a 6.4-mm-diameter by 76$\mathrm{mm}$-long X-axis rod of BLO: $\mathrm{Nd}^{3+}$ has been achieved for the $1.079 \mu \mathrm{m}$ transition, while an yttrium-aluminum garnet doped with $\mathrm{Nd}^{3+}$ (YAG:Nd rod) of the same size in the same system yielded $160 \mathrm{~mJ}$ [1]. The BLO: $\mathrm{Nd}^{3+}$ laser operates well in picosecond pulse mode at a pulse duration of 3$5 \mathrm{ps}$ with an efficiency several times higher than that for the YAG: $\mathrm{Nd}^{3+}$ laser $[2,3]$. Photon detector based upon an Ceactivated lanthanum beryllate scintillator has been developed and patented in [4]. This has stimulated numerous studies of lanthanum beryllate. Let us discuss the main characteristics of the optical material.

Physical properties of BLO were systematized in [5]. In particular, Ref. [5] specifies the following physical parameters: molecular weight of 375.8 , specific gravity of $6.061 \mathrm{~g} / \mathrm{cm}^{3}$, the melting point of $1361^{\circ} \mathrm{C}$, and the Mohs hardness of 6.5. Despite the fact that these properties are not directly related to the subject of the present research work, but they give a comprehensive picture of the boundaries and limitations for the potential applications of BLO single crystals in such important practical areas as laser technology and ionizing radiation detectors. In this regard, they have to be

\footnotetext{
* Corresponding author: i.n.ogorodnikov@urfu.ru
}

mentioned.

From the crystallographic viewpoint, BLO single crystals possess the monoclinic crystallographic system (space group symmetry $C 2 \bar{c}$ ). The number of distinct elements is $Z=4$. The lattice parameters of the unit cell are as follows: $a=753.56 \mathrm{pm}, b=734.76 \mathrm{pm}, c=743.87 \mathrm{pm}, \beta=91.33^{\circ}$, $V_{\mu}=411.7 \times 10^{6} \mathrm{pm}^{3}[6]$. The crystal lattice framework is formed by a continuous spatial network of weakly-distorted $\left[\mathrm{BeO}_{4}\right]$ tetrahedra. The distance between beryllium and lone oxygen atom is significantly shorter than the other three $\mathrm{Be}-$ $\mathrm{O}$ bonds in the tetrahedron. Three-dimensional network of corner-shared tetrahedra $\left[\mathrm{BeO}_{4}\right]$ can be described by the general formula $\mathrm{Be}_{2} \mathrm{O}_{5}$. Each lanthanum ion is surrounded by five oxygen ions, and it takes an $C_{1}$ symmetry site in the void of this network [7].

From the viewpoint of optical properties, BLO represents a biaxial crystal with transparency range from 220 to $5500 \mathrm{~nm}$. The refraction indices in polarized light $(0.6-2.0 \mathrm{~nm})$ were measured by Jenssen et al. (1973) [1]. Dispersion of the refraction indices obeys the Sellmeier law [8]

$$
n^{2}=1+A \lambda^{2} /\left(\lambda^{2}-B\right)
$$

where $\lambda$ is wavelength in $\mu \mathrm{m}$; the Sellmeier coefficients $A$ and $B$ are listed in Tab. 1. From Tab. 1 it follows that the refractive indices are $n_{x}=1.9641, n_{y}=1.9974, n_{z}=2.0348$ at a wavelength of $\lambda=1 \mu \mathrm{m}$.

Electronic energy structure and valence band features of lanthanum beryllate were studied in [9]. The 1-MHz dielectric constants and loss factors of BLO single crystals were determined in [10]. Experimental evaluation of the threshold energy $\left(E_{\mathrm{g}}\right)$ for interband transitions has been performed in [11] on the basis of thermoluminescence (TL) excitation spectra. From [11] it follows that $E_{\mathrm{g}} \approx 6.2-6.5 \mathrm{eV}$ at 
Table 1. Sellmeier coefficients for $\mathrm{La}_{2} \mathrm{Be}_{2} \mathrm{O}_{5}$ single crystals at $290 \mathrm{~K}[8]$.

\begin{tabular}{lcc}
\hline Polarization & $A, \mu \mathrm{m}^{-1}$ & $B, \mu \mathrm{m}^{2}$ \\
\hline$n_{x}$ & 2.7990 & 0.01875 \\
$n_{y}$ & 2.9268 & 0.01918 \\
$n_{z}$ & 3.0725 & 0.01950 \\
\hline
\end{tabular}

$T=80 \mathrm{~K}$. It is worth noting that the TL excitation spectra methodology is described in [12,13] and from general considerations it is evident that this methodology may underestimate the $E_{\mathrm{g}}$ value because of the influence of excitonic or defect states which appear just below $E_{\mathrm{g}}$. In this connection, a more accurate determination of $E_{\mathrm{g}}$ is necessary. Potential for practical application of BLO crystals in laser technology and scintillation detectors, also stimulated research works in the field of crystal growing technology and spectroscopy of rare-earth ions. Let us briefly mention early research works on the study of crystallization processes for the Czochralski grown BLO single crystals $[5,14,15]$. There are several works on the spectroscopy of BLO crystals doped with trivalent rare earth ions: $\mathrm{Nd}^{3+}[5,14,16], \mathrm{Ce}^{3+}$ [17-19], $\mathrm{Pr}^{3+}$ [20-22], and $\mathrm{Tm}^{3+}$ [15].

From the above brief review it is evident that there is a lack of many important optical and electronic properties of BLO single crystals. In particular, there is no reliable data on the properties of the low-energy tail of the host absorption; the bandgap needs more accurate assessment; dispersions of the optical functions are unknown. All these data are essential for the use of BLO host lattice as an optical material for various practical applications.

The aim of this work was to investigate the optical and electronic properties of lanthanum beryllate single crystals in the energy range of the low-energy tail of the host absorption by the means of the optical spectroscopy techniques, including the optical absorption spectroscopy ( $T=80$ and $320 \mathrm{~K})$ and low-temperature $(T=10 \mathrm{~K})$ reflection spectroscopy (4.9$33 \mathrm{eV}$ ) upon excitation by synchrotron radiation. Processing of the experimental data was carried out using the methods of both the dispersion analysis and Kramers-Krönig transforms.

\section{Experimental details}

All the examined lanthanum beryllate single crystals were grown by V. N. Matrosov using the Czochralski method at the Institute of Geology and Geophysics of Siberian Branch of RAS (Novosibirsk, Russia). Crystal growth details have been described in [23]. The grown crystals were inspected by $\mathrm{X}$-ray techniques, and results of these studies have been published previously as separate papers. It is worth noting several few important papers in this series. Tsvetkov et al. [23] have studied BLO single crystals using methods of transmission X-ray topography, X-ray powder diffraction, and X-ray microprobe analysis. The main conclusion of Ref. [23] was formulation of the conditions for growing BLO single crystals having a minimum quantity of structural defects. We do not plan to discuss here the details of Ref. [23], but we would like to mention two important facts. Firstly, all the crystals re- ported in our study are grown under the conditions formulated in Ref. [23]. Secondly, crystallographic parameters of the grown crystals are in agreement with [6]. There is available past paper reported the study of X-ray photoelectron spectra (XPS) for BLO single crystals [9]. We will discuss the XPSresults in the next sections.

Samples in the form of the polished optical quality discs (diameter of $\oslash 10-15 \mathrm{~mm}$, the thickness of $l=0.3-1.0 \mathrm{~mm}$ ) were prepared for spectroscopic studies.

One of the goals of this study is to obtain the spectra of optical functions using the Kramers-Krönig transforms. This procedure requires the experimental reflection spectra recorded in the spectral range as wide as possible. Fortunately, there is a synchrotron radiation spectroscopy that provides such an experimental tool. The reflection spectra at an angle of incidence of $17^{\circ}$ were measured in the energy range of 4$33 \mathrm{eV}$ at the SUPERLUMI station of HASYLAB (Hamburg, Germany) under selective optical excitation by synchrotron radiation [24]. Two in situ interchangeable gratings of the primary monochromator, $\mathrm{Al}$ and $\mathrm{Pt}$ coated, were applied in measurements for low and high energy range, respectively. The resolution of the primary monochromator was typically $0.32 \mathrm{~nm}$. The measurements were performed at a temperature of $8-10 \mathrm{~K}$ with a He-flow cryostat cryostat providing a vacuum of not worse than $5 \times 10^{-8} \mathrm{~Pa}$. A photomultiplier XP2230B (Valvo) with a sodium salicylate coated window was used as a registration system. It should be noted that the experimental spectra were recorded initially in particular units as usual. In most cases it is enough to discuss them. The main objective of this work is related to the calculation of optical functions on the basis of spectroscopic data. In this connection, the experimental reflection spectra required careful preparation to be suitable for use in the calculations. Details can be found elsewhere, see for example [25]. We have carried out calibration of the experimental spectra by the Fresnel equations. During processing of these spectra, we used the known dispersions for refractive index in the transparency region of the crystals $[1,8]$. As a result, the experimental reflection spectrum was presented in terms of the intensity reflection coefficient or reflectance $R=I / I_{0}$, where $I_{0}$ and $I$ are the incident and reflected light intensities, respectively. In the other words, $R$ is the fraction of incident light intensity that is reflected at the crystal surface. It is worth noting that the reflectance $(R)$ is related to the complex amplitude reflection coefficient $(r)$ by the formula $R=|r|^{2}$ (see details, for example, in [25]).

Determination of electronic properties (in particular, bandgap $E_{\mathrm{g}}$ ) from the spectra of the optical functions requires experimental data on the low-energy tail of the hostabsorption of BLO crystal. Optical absorption spectra at 80 and $320 \mathrm{~K}$ were measured at the laboratory of Solid State Physics of Ural Federal University (Yekaterinburg, Russia) by the means of a He $\lambda$ ios Alpha 9423UVA1002E spectrophotometer $(\lambda=190-1000 \mathrm{~nm})$ equipped with the Vision 32 software. The experimental optical absorption coefficient $\alpha_{\exp }$ was determined using the formula $\alpha_{\exp }=-\ln (\tau) / l$, where $\tau$ is the optical transmission coefficient. 


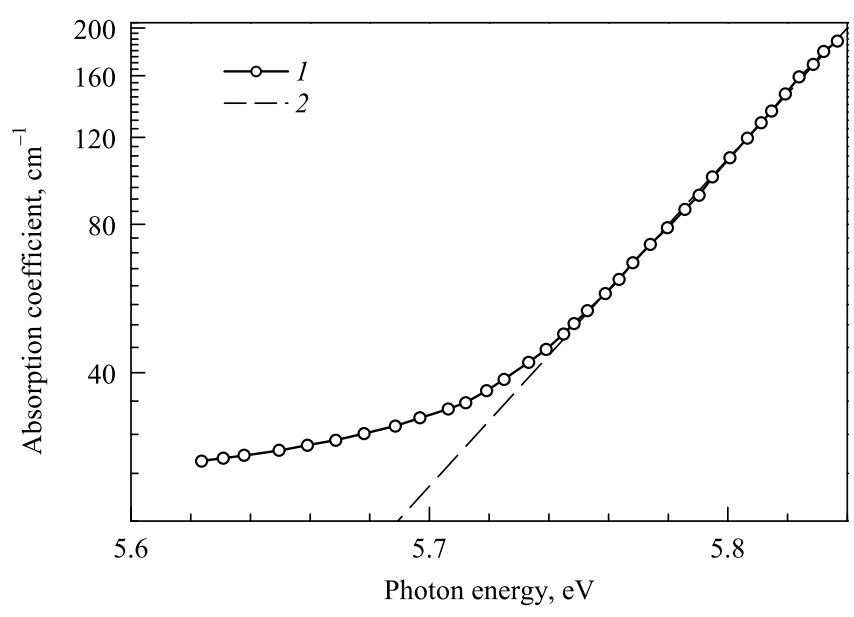

Fig. 1. Absorption spectra of BLO single crystal recorded at $T=80 \mathrm{~K}$ in the energy range of low-energy tail of the host absorption: (1) experimental data $\alpha_{\exp }(E)$; (2) straight line approximation corresponds to the Urbach's tail $\alpha(E)$ of the host absorption.

\section{Experimental results and discussion}

\section{A. Low-energy tail of the host absorption}

Figure 1 shows the optical absorption spectrum of BLO single crystal recorded at $T=80 \mathrm{~K}$ in the energy range of the lowenergy tail of the host absorption. It is known [26, 27] that an exponentially increasing absorption edge in a number of insulators including ionic crystals, semiconductors, and organic crystals follows the empirical expression - the UrbachMartienssen rule [26, 27]

$$
\alpha(E)=\alpha_{0} \exp \left[\frac{\sigma}{k_{\mathrm{B}} T}\left(E-E_{0}\right)\right],
$$

where $\alpha_{0}$ and $E_{0}$ are characteristic parameters of the material, $\sigma$ is the steepness parameter, $k_{\mathrm{B}}$ is the Boltzmann constant, and $T$ is the temperature. Expression (2) in a semilogarithmic coordinate system is represented by a straight line with a slope $a$ and intersect $b$

$$
\begin{aligned}
\log \alpha & =b+a E ; \\
a & =\frac{\sigma \log e}{k_{\mathrm{B}} T} ; \\
b & =\log \alpha_{0}-a E_{0},
\end{aligned}
$$

here we use the notations: $\log =\log _{10}$ is common logarithm, and $e$ is the base of the natural logarithm. From Fig. 1 it is clear that dramatic monotonic increase of the absorption coefficient occurs at energies $E>5.74 \mathrm{eV}$. Optical absorption spectrum comprises two energy regions of different origins. The low-energy region $(E<5.71 \mathrm{eV})$ is dominated by processes which, in our opinion, originated from unidentified lattice defects. The high-energy region $(E>5.74 \mathrm{eV})$ in a semi-logarithmic coordinate system can be approximated by a straight line (Fig. 1, plot 2), which is well comparable with the low-energy Urbach 'tail' of the host absorption. In this connection, the experimental absorption coefficient $\alpha_{\exp }$ (Fig. 1, plot 1) can be represented as a sum of two contributions from the defect related $\left(\alpha_{1}\right)$ and host $(\alpha)$
Table 2. The straight line approximation for the low-energy tail of the host absorption of $\mathrm{La}_{2} \mathrm{Be}_{2} \mathrm{O}_{5}$ single crystals at $T=80$ and $320 \mathrm{~K}$ : slope $(a)$, intersect $(b)$, and the cut-off energy $E_{\mathrm{c}}$ in accordance of the expression (Eq. 3).

\begin{tabular}{cccc}
\hline Temperature, $\mathrm{K}$ & $a$ & $b$ & $E_{\mathrm{c}}, \mathrm{eV}$ \\
\hline 80 & 6.661 & -36.597 & 5.49 \\
320 & 5.184 & -27.217 & 5.25 \\
\hline
\end{tabular}

absorption processes: $\alpha_{\exp }=\alpha_{1}+\alpha$. Table 2 shows the approximation parameters (slope $a$, and intersect $b$ ) for the $\alpha$ process in a semi-logarithmic coordinate system. Fig. 1 (plot 2) shows the $\alpha$ process as a dash line. We defined a cutoff energy $E_{\mathrm{c}}$ at which the absorption coefficient $\alpha$ was equal to $1 \mathrm{~cm}^{-1}$. Energy region below $E_{\mathrm{c}}$ corresponds to the transparency band of the crystal. However, the graphical determination of $E_{\mathrm{c}}$ from Fig. 1 is impossible, because the $E_{\mathrm{c}}$ value is expected in the energy range where $\alpha_{1}$-process dominates the experimental absorption spectrum $\alpha_{\text {exp }}$. Therefore, we have derived the $E_{\mathrm{c}}$ value from approximation parameters for the straight line that fit $\alpha$ in semi-logarithmic coordinate system, Tab. 2 . It is easy to see that $E_{\mathrm{c}}=-b / a=5.49 \mathrm{eV}$ at $T=80 \mathrm{~K}$. The same measurements were carried out above room temperature. These results are not shown in Fig. 1, but the approximation parameters are listed in Tab. 2. From Tab. 2 it follows that $E_{\mathrm{c}}=5.25 \mathrm{eV}$ at $T=320 \mathrm{~K}$. Relative temperature shift of the energy position of low-energy tail of the host absorption resulted in $\Delta E_{\mathrm{c}} / \Delta T=1 \times 10^{-3} \mathrm{eV} / \mathrm{K}$. Writing the equation (Eq. 3 ) for two different temperatures (Tab. 2), we solved a system of equations and find the intersection point for the straight lines with coordinates $E_{0}=6.25-$ $6.30 \mathrm{eV}$ and $\alpha_{0}=(4 \div 5) \times 10^{5} \mathrm{~cm}^{-1}$.

The temperature dependence of the steepness parameter $\sigma$ obeys the expression [27]

$$
\sigma=\sigma_{0} \frac{2 k_{\mathrm{B}} T}{E_{\mathrm{eff}}} \tanh \left(\frac{E_{\mathrm{eff}}}{2 k_{\mathrm{B}} T}\right)
$$

where $\sigma_{0}$ and $E_{\text {eff }}$ are constants. The constant $\sigma_{0}=0.44$ for BLO single crystals have been reported in [17]. Assuming $\sigma_{0}=0.44$ [17] and putting other data from Tab. 2 into the formula (Eq. 4) we can estimate the value of constant $E_{\text {eff }}=57-58 \mathrm{meV}$. This parameter corresponds to the energy of the phonon vibrations interacting with electronic excitations, and its value (57-58 meV) can be tentatively compared with the optical phonons, caused by vibrations of the beryllium sublattice.

A typical feature of BLO single crystals is the lack of excitonic structure in both the absorption and reflection spectra even at low temperatures. Figure 2 illustrates this statement for the reflection spectrum recorded at $T=10 \mathrm{~K}$. According to the theory of Sumi [28], the lack of a characteristic excitonic structure in the reflection spectrum of lanthanum beryllate at low temperature (Fig. 2) and a relatively low value of one of the Urbach rule parameters $\left(\sigma_{0}=0.44\right.$ [17]) may indicate a case of strong exciton-phonon interaction. Based on these arguments, we believe that the low-energy tail of the BLO host absorption corresponds to the absorption of local- 


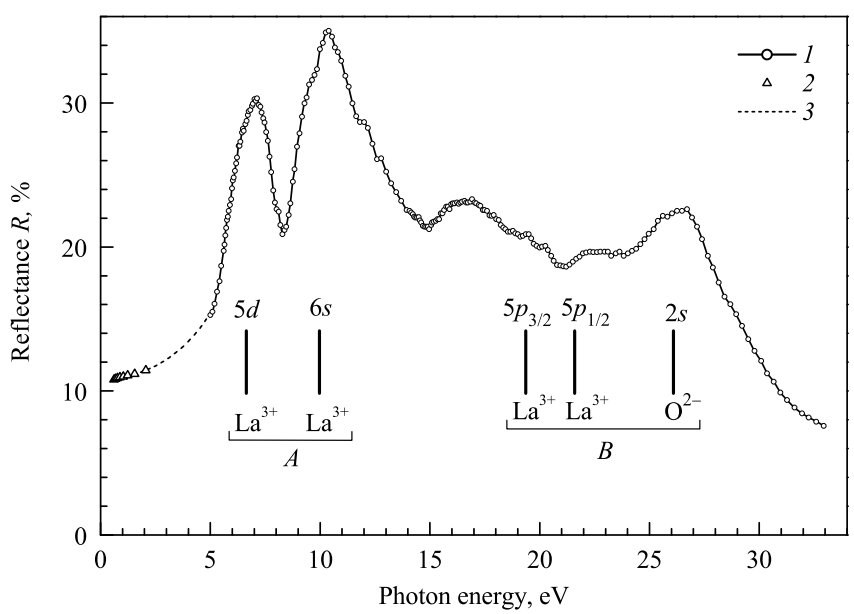

Fig. 2. Reflection spectra: (1) experimental data for BLO single crystal recorded at $10 \mathrm{~K}$, the experimental points are connected by straight lines for better viewing; (2) points in the energy range of $0.6-2.0 \mathrm{eV}$ were calculated using refraction coefficients, taken from [1]; Smooth curve in the energy range of $0.6-4.9 \mathrm{eV}$ was calculated using Sellmeier equation, taken from [8] - (3). Energy positions of the predicted electronic transitions were shown in accordance with [9]. There are transitions: $(A)$ from the valence band top onto the specified levels, and $(B)$ from the specified levels onto the conduction band bottom.

ized excitons in the case of a strong interaction with phonons. It should be noted that a typical value of $\sigma_{0} \approx 0.8$ [27] in alkali-halide crystals is significantly larger than the $\sigma_{0}$ value for lanthanum beryllate. However, such a low value of $\sigma_{0}$ is not exclusive. The value of $0.2 \leq \sigma_{0} \leq 0.63$ have been reported for the crystalline and vitreous $\mathrm{SiO}_{2}$ in dependence on the experimental conditions and the concentration of impurities in the samples [29].

\section{B. Low-temperature reflection spectrum}

Fig. 2 shows the low temperature $(T=10 \mathrm{~K})$ reflection spectrum recorded for BLO single crystal in the energy region from $4.9 \mathrm{eV}$ to $33 \mathrm{eV}$ (about $5 E_{\mathrm{g}}$ ). In the energy region below $4.9 \mathrm{eV}$ (the transparency region of the crystal, extinction index $k=0$ ), the reflection coefficient values $R$ have been obtained on the basis of published data for the refraction indices $n$ using a known formula (see e.g. [30])

$$
R=\frac{(n-1)^{2}+k^{2}}{(n+1)^{2}+k^{2}} .
$$

The refraction indices were obtained from two sources: the experimental data for the energy region of $0.6-2.0 \mathrm{eV}$ were taken from [1] (Fig. 2, plot 2), in the energy range of 0.6$4.9 \mathrm{eV}$ we used the Sellmeier equation (Eq. 1), which was taken from Ref. [8], Fig. 2, curve 3. Experimental reflection spectra were originally recorded in arbitrary units corresponding to the registration system. Subsequent normalization procedure was used to harmonize at $4.9 \mathrm{eV}$ our experimental reflection spectra and calculated $R$ values taken from the literature, Fig. 2.
It should be noted that the reflection spectrum (Fig. 2) differs from the previously published reflection spectra of lanthanum beryllate. Reflection spectra in Ref. [11] were recorded at 80 and $300 \mathrm{~K}$ with lower spectral resolution and therefore they contain fewer structural features. Reflection spectrum from Ref. [17] was registered at $T=10 \mathrm{~K}$ with better spectral resolution, but the spectrum is shown in arbitrary units and is intended only for the analysis of photoluminescence excitation spectra.

Reflection spectrum (Fig. 2) was recorded in a broad energy range and it comprises three groups of bands in the energy ranges of 5-7, 7-12 and 20-27 eV. The lowest-energy bands are located in the vicinity of the fundamental absorption edge, and they correspond to excitonic transitions and they include probably the energy threshold for interband transitions. In the medium-energy region, the bands are due to electronic transitions from the valence band (VB) to the conduction band (CB), i.e. interband transitions. An increase at the reflection coefficient at $E>15 \mathrm{eV}$ corresponds to transitions for both: the transitions onto the highly excited VB states as well as to the excitations of $5 p$-lanthanum states that contribute to the reflection spectrum in the energy region of $21-26 \mathrm{eV}$. Such a spectrum structure is well known for three-fluorides of rare earth metals and it may be correlated with excitons associated with the excitation of the $5 p$-lanthanide levels [31].

Betenekova et al. (1983) [9] have studied experimentally the X-ray photoelectron spectra, and has conducted the MO LCAO calculations of the electronic structure for lanthanum beryllate single crystals. The results of Ref. [9] and significant similarity of our data with the reflection spectra for three-lanthanum fluorides [32] allows us to attribute the low-energy bands at 7.0 and $10.4 \mathrm{eV}$ to electronic transitions from the valence band top, formed by $2 p$ oxygen states, onto the conduction band bottom, formed by $5 d$ and $6 s \mathrm{La}^{3+}$ states. An increase in the reflection coefficient at energies above $15 \mathrm{eV}$ probably corresponds to electronic transitions from the $2 p$ oxygen states onto the highly excited CB states. The bands at 19.5 and $22 \mathrm{eV}$ were attributed to the excitation of $5 p_{3 / 2}$ and $5 p_{1 / 2} \mathrm{La}^{3+}$ states (possibly cationic excitons). Electronic transitions from $2 s \mathrm{O}^{2-}$ states are responsible for the $26.5 \mathrm{eV}$ peak in the reflection spectrum. Energy level diagram of lanthanum beryllate was constructed on the basis of this interpretation (Fig. 2). It definitely is consistent with the positions of the energy levels in the X-ray photoelectron spectra of BLO crystals [9].

It is worth noting that the the reflection spectra in the energy region of the low-energy tail of the BLO host absorption, demonstrate a complete lack of excitonic transitions originating from the valence band. This makes it difficult to determine the minimum energy for interband transitions $E_{\mathrm{g}}$. Known $E_{\mathrm{g}}$ estimate has been performed on the basis of the excitation spectrum for the low-temperature thermally stimulated luminescence: $E_{\mathrm{g}}=6.2-6.5 \mathrm{eV}$ at $80 \mathrm{~K}$ [11]. On this basis, we believe that the excitons in lanthanum beryllate are metastable and are therefore not recorded in the reflection spectra. They are manifested only in the processes of radiative relaxation of electronic excitation [17]. To determine the $E_{\mathrm{g}}$ value it is necessary to calculate dispersions of the optical 


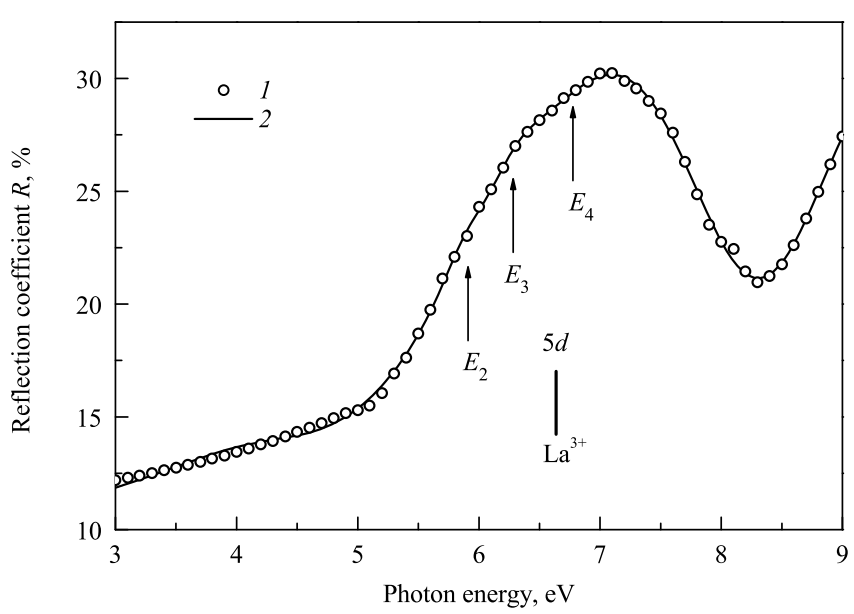

Fig. 3. Reflection spectra in the low-energy tail of the host absorption: (1) Experimental data for BLO crystal composed from two part: our experimental data recorded for single crystal at $10 \mathrm{~K}$ (4.9$9.0 \mathrm{eV})$ and calculated data obtained using Sellmeier equation, taken from [8] $(3.0-4.9 \mathrm{eV}) ;(2)$ result of the approximation. The vertical arrows indicate the transition energies $\left(E_{j}\right)$ calculated for oscillators. Energy positions of the predicted electronic transition from the valence band top onto the specified level of $\mathrm{La}^{3+}$ was shown in accordance with [9].

functions.

\section{C. Optical functions of lanthanum beryllate}

To expand the analyzed energy region in the processing of the reflectance spectra, we added our experimental data (4.9$33 \mathrm{eV})$ by the published data from Ref. [8] $(3-4.9 \mathrm{eV})$ that relate to the transparency range of the crystal. We used two different processing methods: dispersion analysis in the vicinity of the low-energy tail of the host absorption $(3-9 \mathrm{eV})$ at $10 \mathrm{~K}$ (Fig. 3) and Kramers-Krönig analysis in a broad energy region of $3-33 \mathrm{eV}$ at $10 \mathrm{~K}$.

\section{C.1. Dispersion analysis of the reflection spec- trum}

Within the framework of the dispersion model [33, 34], the contribution of each oscillator $j$ in the complex dielectric constant of the optical material is given by the expression

$$
\delta \hat{\varepsilon}_{j}=\frac{M_{j}}{E_{j}^{2}-E^{2}-i \Gamma_{j} E},
$$

where $i$ is the imaginary unit, $E$ is an excitation energy; $E_{j}$, $\Gamma_{j}$, and $M_{j}$ are the spectral parameters of the oscillator: position of the maximum, full width, and amplitude, respectively. Approximation of the reflection spectrum (Fig. 3) has demanded accounting of five oscillators $(j=1-5)$.

$$
\hat{\varepsilon}(E)=\varepsilon_{\infty}+\sum_{j=1}^{5} \frac{M_{j}}{E_{j}^{2}-E^{2}-i \Gamma_{j} E},
$$

where $\varepsilon_{\infty}$ is a high-frequency dielectric constant.

Complex refractive index $\hat{n}$ is associated with the dielectric constant through the relation $\hat{\varepsilon}=\hat{n}^{2}$. Hire $\hat{n}=n+i k$,
Table 3. Parameters of approximation for reflection spectra recorded at $T=10 \mathrm{~K}$ in the energy range of the low-energy tail of the host absorption: ordinal number of a transition $(j)$, transition energy $\left(E_{j}\right)$, full width at half maximum $\left(\Gamma_{j}\right)$ and amplitude $\left(M_{j}\right)$

\begin{tabular}{cccc}
\hline Oscillator & $E_{j}$ & \multicolumn{1}{c}{$M_{j}$} & $\Gamma_{j}$ \\
\hline 1 & 4.453 & 6.09 & 2.00 \\
2 & 5.911 & 1.90 & 0.51 \\
3 & 6.282 & 8.16 & 0.82 \\
4 & 6.775 & 51.61 & 1.85 \\
5 & 8.792 & 19.91 & 1.61 \\
\hline$\varepsilon_{\infty}$ & & 1.447 & \\
\hline
\end{tabular}

Note. Parameters $E_{j}$ and $\Gamma_{j}$ are given in electron-volts.

where $n$ and $k$ are the refraction and extinction indices, respectively. For a given angle of incidence $\theta$, the reflection coefficient for the $s$-polarized radiation in a vacuum can be calculated by the formula [30]

$$
R(E)=\left|\frac{\cos \theta-\hat{n} \cos \hat{\theta}}{\cos \theta+\hat{n} \cos \hat{\theta}}\right|^{2} .
$$

Complex refraction angle $\hat{\theta}$ is determined by the Snell's law $\sin \theta=\hat{n} \sin \hat{\theta}$. Approximation quality for the experimental data was estimated by the formula

$$
D=\frac{1}{N} \sum_{j=1}^{N} \frac{\left|R_{m j}-R_{c j}\right|}{R_{m j}},
$$

where $R_{m j}$ and $R_{c j}$ are the measured $(m)$ and calculated $(c)$ reflection spectra, $N$ is the number of experimental points. The best fit results are presented in Tab. 3 and in Fig. 3. They correspond to $D=0.0075$. Let's discuss a possible interpretation of the electronic transitions.

Oscillator with the index $j=1\left(E_{1}\right)$ is located in the transparency region of the crystal (Tab. 3) and takes into account the contributions from all oscillators located in the lowenergy region beyond the recorded spectrum. Oscillator with the index $j=5$ is located in the host absorption region and includes the contributions from all oscillators located in a highenergy region outside the recorded spectrum. In that regard, oscillators, $j=1$ and 5 can not be associated with the specific electronic transitions.

In contrast, the oscillators $j=2-4$ (Tab. 3 ) are located in the energy range of the recorded spectrum, including the energy range of the low-energy tail of the host absorption. The highest amplitude oscillators can be associated with the maximum of the excitonic absorption $\left(E_{3}=6.28 \mathrm{eV}\right)$ and the energy threshold for interband transitions $E_{\mathrm{g}}\left(E_{4}=6.78 \mathrm{eV}\right)$. Indeed, the parameter $E_{0}$, resulting in the Urbach rule for many optical materials, is usually located near the energy position of the exciton absorption peak [27]. The $E_{\mathrm{g}}$ energy usually exceeds the energy position of the excitonic absorption peak for a few tenths of an electron volt. It is worth noting two facts. First, our estimate of $E_{\mathrm{g}}=6.78 \mathrm{eV}$ is close to the calculated data [9], where the energy gap between the highest 
occupied molecular orbitals ( $2 p$ states of $\left.\mathrm{O}^{2-}\right)$ and lowest vacant molecular orbitals ( $5 d$ states of $\mathrm{La}^{3+}$ ) was about $6.69 \mathrm{eV}$. Betenekova et al. (1983) [9] have found that the lowestenergy electronic excitations in lanthanum beryllate was due to electronic transitions between $2 p \mathrm{O}$ and $5 d$ La states of the host ions. Second, our assessment of $E_{\mathrm{g}}=6.78 \mathrm{eV}$ gives a slightly higher value of the energy threshold for interband transitions than $E_{\mathrm{g}} \simeq(6.2-6.5) \mathrm{eV}$, derived from the lowtemperature TL excitation spectrum in Ref. [11]. However, our assessment of threshold for interband transitions do not contradict with the experimental TL excitation spectrum represented in Ref. [11]. Indeed, our value $E_{\mathrm{g}}=6.78 \mathrm{eV}$ corresponds exactly to the energy position of the dominant lowenergy peak in the TL excitation spectrum [11]. Origin of low-intensity oscillator $E_{2}=5.9 \mathrm{eV}$ at this stage is unknown. On the one hand, its energy corresponds to the host absorption range of a crystal. Therefore, its origin may be due to the inhomogeneity of the spatial structure and the differences in the polarization of the electronic transitions. On the other hand, its energy position is very close to the fundamental absorption edge of the crystal and its origin may be due to the localization of electronic excitations near the unidentified defects.

\section{C.2. Kramers-Krönig analysis of the reflection spectrum}

Figs. 4 and 5 show the calculated results for the dispersions of the optical functions carried out using the Kramers-Krönig transforms. We used the difference Kramers-Krönig method to calculate the optical functions [25]. Experimental data on the low-temperature $(T=10 \mathrm{~K})$ reflection spectra of BLO single crystals were used in these calculations (Fig. 4).

The calculated values of the refraction index $n$ (Fig. 4) in the energy region of the optical transparency of the crystal coincide well with both the experimental data obtained in Ref. [1] for the energy region of $0.6-2.0 \mathrm{eV}$, and the calculated values obtained for the energy range of $0.6-4.9 \mathrm{eV}$ obtained using the Sellmeier equation [8]. This may be regarded as evidence of the correctness of the calculation performed for the optical functions of lanthanum beryllate. The lowest energy peak of $n(E)$ is located at $6.3 \mathrm{eV}$, the next $n(E)$ peak is observed at $8.9 \mathrm{eV}$. Refractive index $n=1$ is located at $E=12.8 \mathrm{eV}$. Minimum of the refractive index is observed at $27 \mathrm{eV}$. With further increase in energy, $n(E)$ value is monotonically increasing and tends asymptotically to the unit value.

A monotonic increase of $k(E)$ starts with an energy of about $E=5.8 \mathrm{eV}$, and reaches the lowest energy maximum at $7.4 \mathrm{eV}$. Next dominant maximum of $k(E)$ is located at $10.1 \mathrm{eV}$. Further, the $k(E)$ value gradually decreases in the entire measured energy range up to $33 \mathrm{eV}$, Fig. 4.

Dispersion curves for $\varepsilon_{1}(E)$ and $\varepsilon_{2}(E)$ follow principally, the $n(E)$ and $k(E)$ spectra. The lowest energy dominant maximum of $\varepsilon_{1}(E)$ is located at $6.3 \mathrm{eV}$. Energy positions of the $\varepsilon_{2}(E)$ maxima are observed at $E=7.1$ and $9.4 \mathrm{eV}$ and they are slightly displaced in comparison with the $k(E)$ peak positions, Fig. 4.

Broad peaks at $21-24 \mathrm{eV}$ and $25-28 \mathrm{eV}$ in the reflection spectrum correspond to the most intense peaks in the

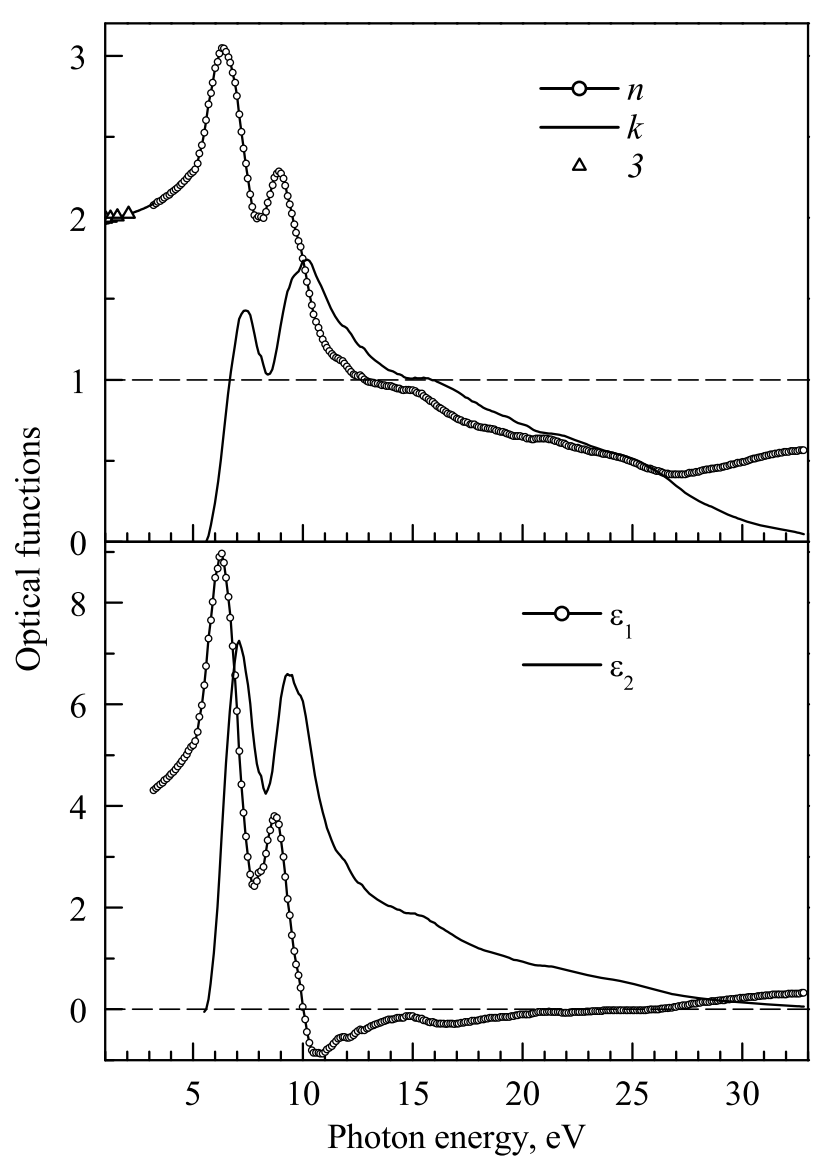

Fig. 4. Dispersions of the optical functions calculated using the Kramers-Krönig transform. Refraction coefficients for the energy range of 0.6-2.0 eV were taken from [1] - (3). Smooth curve in the energy range of $0.6-4.9 \mathrm{eV}$ was calculated using Sellmeier equation, taken from [8].

$-\operatorname{Im}(1+\hat{\varepsilon})^{-1}$ and $-\operatorname{Im} \hat{\varepsilon}^{-1}$ spectra. It is known [35] that under certain conditions, the energy positions of the most intense peaks in these spectra coincide with the energies of the surface $\left(E_{\mathrm{ps}}\right)$ and bulk $\left(E_{\mathrm{pv}}\right)$ plasmons. Our calculation for the lanthanum beryllate single crystal at $10 \mathrm{~K}$ led to the following results: $E_{\mathrm{ps}}=(21.7-24.5) \mathrm{eV}$ and $E_{\mathrm{pv}}=27.5 \mathrm{eV}$.

The absorption coefficient $\mu(E)$ (Fig. 5) was calculated on the basis of the dispersions of the optical functions (Fig. 4) using the formula

$$
\mu=\frac{4 \pi k}{\lambda} \times 10^{7}
$$

where $\mu$ is the absorption coefficient, $\mathrm{cm}^{-1}, \lambda$ is wavelength, $\mathrm{nm}$. The $\mu(E)$ spectrum provides the basis for future analysis of the excitation spectra of intrinsic and extrinsic photoluminescence in lanthanum beryllate single crystals.

\section{Conclusions}

The present paper reports the results of a study on the optical and electronic properties of beryllate lanthanum single crystals carried out in the energy region of the low-energy tail of the host absorption of the crystal using optical spectroscopy 


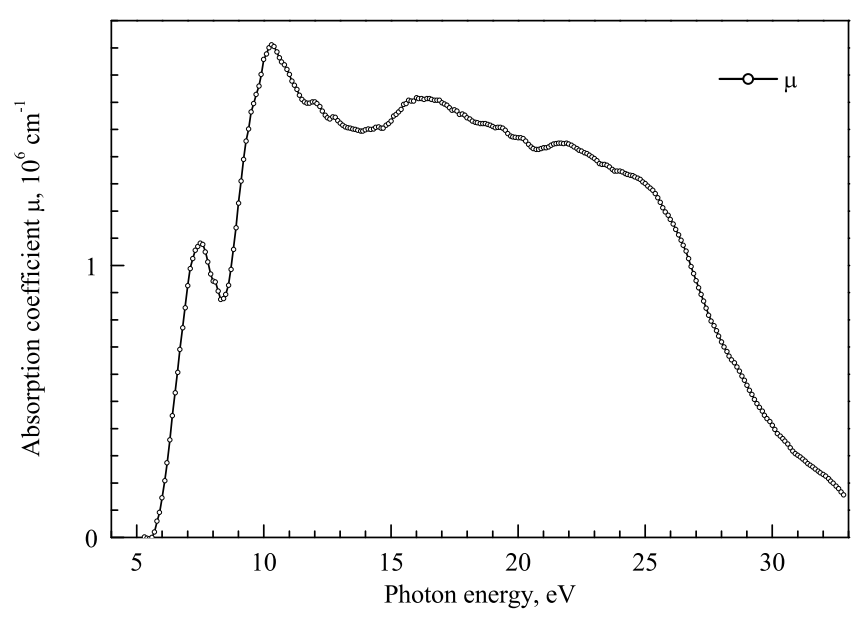

Fig. 5. Dispersion of the optical function $\mu(E)$ calculated using the Kramers-Krönig transform.

techniques, including optical absorption spectroscopy ( $T=80$ and $320 \mathrm{~K})$ and low temperature $(T=10 \mathrm{~K})$ reflection spectroscopy in a broad energy range from 4.9 to $33 \mathrm{eV}$ upon excitation with the synchrotron radiation. To analyze the experimental data we used methods of both the dispersion analysis and Kramers-Krönig transforms. The main findings are as follows.

1. It was revealed that above the cut-off energy $E_{\mathrm{c}}=5.49$ (at $80 \mathrm{~K}$ ) and $5.25 \mathrm{eV}$ (at $320 \mathrm{~K}$ ) there is a monotonic exponential increase in the optical absorption coefficient, which is comparable with a low-energy tail of the host absorption. These values of $E_{\mathrm{c}}$ can be taken as an estimate of the blue border of the optical transparency region for lanthanum beryllate single crystals.

2. Analysis of the experimental data on the low-energy tail of the host absorption made within the Urbach-Martiensen theory, allowed us to determine the Urbach's rule parameters: $E_{0}=6.25 \div 6.30 \mathrm{eV}$ and $\alpha_{0}=(4 \div 5) \times 10^{5} \mathrm{~cm}^{-1}$. Additional consideration of the $\sigma_{0}=0.44$ value from Ref. [17] let us on the basis of our experimental data to assess the value of $E_{\text {eff }}=57 \div 58 \mathrm{meV}$.

3. It was found that the observed properties of the lowenergy tail of the host absorption (no characteristic exciton structure in the reflection spectrum at low temperature and relatively low value of one of the parameters of the Urbach rule: $\sigma_{0}=0.44$ [17]) indicate the case of strong excitonphonon interaction. The origin of the low-energy tail of the host absorption in lanthanum beryllate was tentatively ascribed to the absorption of localized excitons in the case of a strong interaction with phonons. Such excitons are metastable and are therefore not recorded in the reflection spectra. Within the framework of the dispersion analysis of the reflection spectra, we calculated the energy position of the exciton absorption maximum $E_{\mathrm{ex}}=6.28 \mathrm{eV}$, and the threshold for interband transitions $E_{\mathrm{g}}=6.78 \mathrm{eV}$.

4. Analysis of the experimental reflection spectra together with the calculated data on the electronic structure of BLO taken from Ref. [9], allowed us to interpret the most in- tense bands in the reflection spectrum of BLO single crystals. The 7.0 and $10.4 \mathrm{eV}$ bands were attributed to electronic transitions from the VB top states $(2 p \mathrm{O})$ onto the $\mathrm{CB}$ bottom states ( $5 d \mathrm{La}$ and $6 s \mathrm{La}$ ); reflection at $E>15 \mathrm{eV}$ has been attributed to such transitions onto highly excited CB-states. The 19.5, 22 and $26.5 \mathrm{eV}$ bands were attributed to electronic transitions from deep levels $\left(5 p_{3 / 2} \mathrm{La}, 5 p_{1 / 2} \mathrm{La}\right.$, and $2 s$ $\mathrm{O})$ onto the CB-bottom. Such an interpretation of the BLO reflection spectrum does not exclude that the 19.5 and $22 \mathrm{eV}$ bands correspond to the excitation of the cationic $\left(\mathrm{La}^{3+}\right) \mathrm{ex}-$ citons.

5. By means of the Kramers-Krönig transforms, we found the dispersions of the optical functions $\hat{n}(E), \hat{\varepsilon}(E)$ and $\mu(E)$ for the energy range of $3-33 \mathrm{eV}$. The energy positions of the most intense peaks in the spectra of $-\operatorname{Im}(1+\hat{\varepsilon})^{-1}$ and $-\operatorname{Im} \hat{\varepsilon}^{-1}$ at $10 \mathrm{~K}$ were compared with the energies of the surface $\left(E_{\mathrm{ps}}=21.7-24.5 \mathrm{eV}\right)$ and bulk $\left(E_{\mathrm{pv}}=27.5 \mathrm{eV}\right)$ plasmons.

The obtained dispersions of the optical functions provide the basis for further analysis of the excitation spectra for the intrinsic and extrinsic photoluminescence in lanthanum beryllate single crystals.

\section{Acknowledgments}

Authors are grateful to V.N. Matrosov for providing the samples examined, and A. A. Maslakov for assistance in the optical absorption measurements. This work was partly supported by the Ministry of Education and Science of the Russian Federation, Russia (the basic part of the government mandate), and HASYLAB DESY, Germany (Project No.20110843).

\section{References}

[1] H. P. Jenssen, R. F. Begley, R. Webb, and R. C. Moris, "Spectroscopic properties and laser performance of $\mathrm{Nd}^{3+}$ in lanthanum beryllate," J. Appl. Phys. 47, 1496-1500 (1976).

[2] R. Scheps, "Efficient laser diode pumped Nd lasers," Appl. Opt. 28, 89-91 (1989).

[3] R. C. Morris, C. F. Cline, R. F. Begley, M. Dutoit, P. J. Harget, H.P. Jenssen, T. S. LaFrance, and R. Webb, "Lanthanum beryllate: A new rare-earth ion laser host," Appl. Phys. Lett. 27, 444-445 (1975).

[4] J. B. Czirr and M. Berrondo, "Photon detector based upon an activated lanthanide beryllate scintillator," United States Patent 5483062. (09.01.1996). (Mapleton, Orem, UT).

[5] A. A. Kaminskii, T. Ngoc, S.E. Sarkisov, V. N. Matrosov, and M.I. Timoshechkin, "Growth, spectral and laser properties of $\mathrm{La}_{2} \mathrm{Be}_{2} \mathrm{O}_{5}: \mathrm{Nd}^{3+}$ crystals in the ${ }^{4} F_{3 / 2} \rightarrow{ }^{4} I_{11 / 2}$ and ${ }^{4} F_{3 / 2} \rightarrow{ }^{4} I_{13 / 2}$ transitions," Phys. Status Solidi (a) 59, 121$132(1980)$

[6] L.A. Harris and H.L. Yakel, "The crystal structure of $\mathrm{La}_{2} \mathrm{Be}_{2} \mathrm{O}_{5}$," Acta Crystallogr.Section B 24, 672-682 (1968).

[7] E. G. Tulsky and J. R. Long, "Dimensional reduction: A practical formalism for manipulating solid structures," Chem. Mater. 13, 1149-1166 (2001).

[8] M. J. Weber, Handbook of Optical Materials (CRC Press, Boca Raton, London, New York, 2003).

[9] T. A. Betenekova, A. V. Kruzhalov, N. M. Osipova, V. P. Palvanov, V. L. Petrov, and I. N. Shabanova, "Electron structure and valence band structure of beryllium orthosilicate and lanthanum beryllate," Fiz. tverd. tela 25, 175-179 (1983). 
[10] R. D. Snannon, M. A. Sunurvranran, A. N. Mariano, T. E. Gier, and G. R. Rossman, "Dielectric constants of diaspore and B-, $\mathrm{Be}-$, and P-containing minerals, the polarizabilities of $\mathrm{B}_{2} \mathrm{O}_{3}$ and $\mathrm{P}_{2} \mathrm{O}_{5}$, and the oxide additivity rule," Am. Mineral. 77, 101-106 (1992).

[11] A. V. Kruzhalov, V.A. Pustovarov, A. A. Maslakov, V.L. Petrov, and B.V. Shulgin, "Luminescence excitation and reflection spectra of $\mathrm{La}_{2} \mathrm{Be}_{2} \mathrm{O}_{5}$ crystals in the 5-36 eV region," Opt. Spectrosc. 63, 268-270 (1987).

[12] N. Kristianpoller, D. Weiss, N. Khaidukov, V. Makhov, and R. Chen, "Thermoluminescence of some $\mathrm{Pr}^{3+}$ doped fluoride crystals," Radiat. Meas. 43, 245-248 (2008).

[13] R. Chen and V. Pagonis. Thermally and Optically Stimulated Luminescence: A simulation Approach (John Wiley \& Sons, Ltd, 2011).

[14] Y. K. Voronko, G. V. Maksimova, V.V. Osiko, A. A. Sobol, B.P. Starikov, and M. I. Timoshechkin, "Spectroscopic properties of $\mathrm{La}_{2} \mathrm{Be}_{2} \mathrm{O}_{5}: \mathrm{Nd}^{3+}$ single crystals," Phys. Status Solidi (a) 17, K41-K43 (1973).

[15] V. Sudesh, J.A. Piper, E. M. Goldys, and R. S. Seymour, "Growth, characterization, and laser potential of Tm: $\mathrm{La}_{2} \mathrm{Be}_{2} \mathrm{O}_{5}$," J. Opt. Soc. Am. B-Opt. Physics 15, 239-246 (1998).

[16] X. Zhang, X. Zhang, R. Zhu, and G. Wu, "Suitable for LD pumping-crystal $\mathrm{Nd}^{3+}: \mathrm{La}_{2} \mathrm{Be}_{2} \mathrm{O}_{5}$, , in Solid State Lasers $V$, 2698, 210-215 (1996).

[17] V. A. Pustovarov, V.L. Petrov, É. I. Zinin, M. Kirm, G. Zimmerer, and B. V. Shulgin, "Optical and luminescent VUV spectroscopy of $\mathrm{La}_{2} \mathrm{Be}_{2} \mathrm{O}_{5}$ crystals," Phys. Solid State 42, 253-256 (2000).

[18] D. M. Gualtieri, "Cathodoluminescence of $\mathrm{Ce}: \mathrm{La}_{2} \mathrm{Be}_{2} \mathrm{O}_{5}$ single crystals," J. Luminesc. 60-61, 127-130 (1994).

[19] A. V. Kruzhalov, A. A. Maslakov, V.L. Petrov, and B.V. Shulgin, "Temperature features of Ce luminescence yield spectra in lanthanum beryllate," Zh. Prikl. Spektrosk. 45, 859-861 (1986).

[20] N. V. Kuleshov, A.S. Shinkevich, V.G. Shcherbitsky, V.P. Mikhailov, T. Danger, T. Sandrock, and G. Huber, "Luminescence and time-resolved excited state absorption measurements in $\mathrm{Pr}^{3+}$-doped $\mathrm{La}_{2} \mathrm{Be}_{2} \mathrm{O}_{5}$ and $\mathrm{KGd}\left(\mathrm{WO}_{4}\right)_{2}$ crystals," Opt. Mater. 5, 111-118 (1996).
[21] S. Mahlik, M. Malinowski, and M. Grinberg, "High pressure luminescence and time resolved spectra of $\mathrm{La}_{2} \mathrm{Be}_{2} \mathrm{O}_{5}: \mathrm{Pr}^{3+}$, Opt. Mater. 34, 164-168 (2011).

[22] R. Piramidowicz, M. Kowalska, and M. Malinowski, "Energy transfer processes in $\operatorname{Pr}^{3+}: \mathrm{Be}_{2} \mathrm{La}_{2} \mathrm{O}_{5}$ crystals," J. Alloys Compd. 300-301, 430-434 (2000).

[23] E. G. Tsvetkov, G. M. Rylov, and V. N. Matrosov, "Formation of lanthanum beryllate real structure under different crystallization conditions," Mater. Res. Bull. 41, 307-318 (2006).

[24] G. Zimmerer, "SUPERLUMI: A unique setup for luminescence spectroscopy with synchrotron radiation," Radiat. Meas. 42, 859-864 (2007).

[25] V. Lucarini, J. J. Saarinen, K. E. Peiponen, and E. M. Vartiainen, Kramers-Krönig Relations in Optical Materials Research (Springer-Verlag, Berlin, Heidelberg, 2005).

[26] F. Urbach, "The long-wavelength edge of photographic sensitivity and of the electronic absorption of solids," Phys. Rev. 92, 1324-1324 (1953).

[27] H. W. Martienssen, "Über die excitonenbanden der alkalihalogenidkristalle," J. Phys. Chem. Solids 2, 257-267 (1957).

[28] H. Sumi and Y. Toyozawa, "Urbach-Martienssen rule and exciton trapped momentally by lattice vibration," J. Phys. Soc. Jpn. 31, 342-358 (1971).

[29] I. T. Godmanis, A. N. Trukhin, and K. Hübner, "Excitonphonon interaction in crystalline and vitreous $\mathrm{SiO}_{2}$," Phys. Status Solidi (b) 116, 279-287 (1983).

[30] M. Born and E. Wolf, Principles of optics (Pergamon, New York, 1980).

[31] C. Kunz, ed., Synchrotron radiation: Techniques and Applications, $\mathbf{1 0}$ of Topics in current physics (Springer-Verlag, Berlin, Heidelberg, New-York, 1979). 442 p.

[32] C. G. Olson, M. Piacentini, and D. W. Lynch, "Optical properties of single crystals of some rare-earth trifluorides, 5-34 eV," Phys. Rev. B: Cond. Matter 18, 5740-5749 (1978).

[33] M. Weissbluth, Atoms and Molecules (Academic Press, New York, San Francisco, London, 1978).

[34] P. W. Milonni and J. H. Eberly, Laser Physics (John Wiley \& Sons, Inc., Hoboken, New Jersey, 2010).

[35] D. Pines, Elementary excitation in solids (W. A. Benjamin, N.Y.-Amsterdam, 1963). 